\title{
In vivo evidence for the role of Regl in gastric regeneration: transgenic overexpression of Regl accelerates the healing of experimental gastric ulcers
}

\author{
Hiroyuki Fukuhara ${ }^{1}$, Yasunori Kadowaki ${ }^{1}$, Takayuki Ose ${ }^{1}$, Aziz Monowar ${ }^{1}$, Hiroshi Imaoka ${ }^{1}$, Shunji Ishihara ${ }^{1}$, \\ Shin Takasawa ${ }^{2}$ and Yoshikazu Kinoshita ${ }^{1}$
}

On the basis of its temporal and spatial pattern of expression during the healing of gastric ulcers, Regl is implied to be a key growth factor governing the gastric progenitor cell proliferation, which is fundamental for reconstruction of the gastric tissue; however, there is no direct in vivo evidence. The aim of this study was to use Regl-transgenic (Tg) mice to test the role of Regl protein in the healing of experimentally induced gastric ulcers. The stomachs from 48 pairs of wildtype $(\mathrm{Wt})$ and $\mathrm{Tg}$ littermates were examined for gastric erosions after $24 \mathrm{~h}$ of water-immersion stress, or, 6, 12, 18 and $24 \mathrm{~h}$ after oral administration of $\mathrm{HCl} /$ ethanol. Expression levels of $c$-fos and c-myc proto-oncogenes were examined over time by real-time reverse transcriptase PCR to assess gastric cell proliferation. Almost all the littermate pairs tested showed superiority of $\mathrm{Tg}$ mice over Wt mice in the ability of decreasing ulcer index (UI) (cumulative length of erosion). The timecourse study revealed that the Uls of Tg were lower in the healing phase, and not in the injury phase. The fraction of proliferating cells was higher in Tg mice than in Wt mice throughout the time course as assessed by c-fos expression levels. This is the first in vivo evidence that Regl has a role in gastric ulcer healing. We suggest that Regl exerts its effects by promoting growth and not by cytoprotection.

Laboratory Investigation (2010) 90, 556-565; doi:10.1038/labinvest.2010.42; published online 15 February 2010

KEYWORDS: gastric ulcer; Tg mouse; Regl

Gastric ulcer healing is a complicated but finely regulated process, and serves as a good model for studying the molecular mechanism of tissue regeneration. ${ }^{1,2}$ In the early stage of this process, signaling pathways, which transduce proliferation signal from the site of the injury to the gastric progenitor cells, would be engaged. At the end of the pathways would be growth factors that directly interact with the progenitor cells. These factors are particularly important because they initiate and maintain progenitor cell proliferation, which is fundamental for reconstruction of the epithelial portion of gastric tissue; however, there is little information about their molecular nature.

In 1988, RegI was isolated as a growth factor crucial for pancreatic regeneration and was given the name (for regenerating gene). ${ }^{3}$ We previously examined the temporal and spatial patterns of expression of several factors in experi- mental gastric ulcers in the rat, and tested their effects in in vitro culture systems for gastric cells. These studies delineated one of above gastric signaling pathways, at the end of which is located RegI. ${ }^{4-7}$ At the beginning of the pathway, a proinflammatory cytokine CINC2 $\beta$ (the rat counterpart of human interleukin 8) is secreted from the injured gastric mucosal epithelial cells located around the ulcer margin. ${ }^{6,7}$ This factor is then delivered to enterochromaffin-like (ECL) cells, a class of enteroendocrine cells located deep in the basal portion of the gastric gland. In response to the cytokine stimuli, the ECL cells surrounding the ulcerous crater rapidly produce RegI ${ }^{4}$ which moves back upward to interact with the gastric progenitor cells and to trigger their proliferation. ${ }^{5}$ Despite this advance, however, direct in vivo evidence is still lacking for the effect of RegI in the process of gastric ulcer healing.

${ }^{1}$ Department of Gastroenterology and Hepatology, School of Medicine (Formerly Second Department of Internal Medicine, Shimane Medical University), Shimane University, Izumo, Japan and 'Department of Biochemistry, School of Medicine, Nara Medical University, Kashihara, Japan

Correspondence: Dr Y Kadowaki, MD, PhD, Department of Gastroenterology and Hepatology, School of Medicine, Shimane University, 89-1, Enya-cho, Izumo, Shimane 693-8501, Japan.

E-mail: y-kado@momo.so-net.ne.jp

Received 27 April 2009; revised 11 November 2009; accepted 6 December 2009 
Recently, we created RegI-transgenic ( $\mathrm{Tg}$ ) mice and showed that RegI has growth-promoting effects on gastric progenitor cells in the process of 'generation' of gastric mucosal architecture. ${ }^{8}$ Previous reports ${ }^{9,10}$ showed that the mouse stomach, in contrast to the rat stomach, shows very low levels of RegI expression, providing an ideal experimental system with which to test the effects of the regI transgene. Tg mice of 3 weeks old, just finishing the period of gastric gland morphogenesis, showed apparent gastric mucosal hypertrophy and elevated expression of a cell proliferation marker, compared with littermate controls. ${ }^{8}$ Thus, we now have an experimental system in which to test the function of RegI in gastric ulcer healing (gastric mucosal 'regeneration') in vivo. In this study, using Reg-Tg mice, we asked whether the transgene accelerates the healing of experimental gastric ulcers. The data provide the first in vivo evidence that RegI has a crucial role in gastric ulcer healing.

\section{MATERIALS AND METHODS}

\section{Tg Animals}

All studies with mice were approved by the Shimane University ethics committee for animal experimentation. As described in our previous report, ${ }^{8}$ the vector used to express the rat RegI protein was constructed by modifying the plasmid pEGFP-N1 (Promega, Madison, WI, USA), which contains the green fluorescent protein (GFP) gene under the control of the human cytomegalovirus (CMV) immediate early promoter. The CMV promoter region spans from nucleotide number 1 to 589 in the plasmid vector. The enhancer region lies from 59 to 465 , TATA box from 554 to 560 and the transcription start site at 583 . The plasmid was digested by SacII (651) and NotI to remove the GFP sequence, and ligated to the rat regI cDNA fragment obtained by reverse transcriptase PCR from rat stomach RNA. RT-PCR was performed using the following primers that contain the sequence flanking the rat Reg-coding region: 5'-GCAGAGATTGTTGACTTGCA- $3^{\prime}$ and $5^{\prime}$-GATAGATGGTCTAGTTT CAC- $3^{\prime}$. The resultant vector plasmid was digested by AseI and AflII to release the fragment containing the human CMV immediate early promoter, the rat cDNA sequence covering the coding region and the SV40 polyA signal sequence. The fragment was separated by an agarose gel and purified using MinElute Gel Extraction Kit (Qiagen, Tokyo, Japan). RegI-Tg mice $\left(\mathrm{Reg}^{+/-}\right)$were generated by injection of the DNA fragment into fertilized eggs obtained from BDF1 female mice. The progeny mice were bred with BDF1 wild-type (Wt) mice (Nihon Clea, Tokyo, Japan), not with C57BL/6 or DBA, to efficiently obtain a large battery of $\mathrm{reg}^{+/-} \mathrm{Tg}$ and $\mathrm{Wt}$ littermate pairs. All mice were housed in standard cages with 12-h cycles of light and dark, and had ad libitum access to normal mouse chow and water.

\section{Histopathological Analysis}

The protocol for immunohistochemistry is described in our previous report. ${ }^{9}$ The following primary antibodies were used in this study: monoclonal mouse IgG2a anti-mouse proliferating cell nuclear antigen (Santa Cruz Biotechnology) (1:1000) and monoclonal mouse IgG2a anti-rat RegI antibody (1:50). ${ }^{11}$ For double staining of Tg RegI protein and chromogranin A, the tissue slides were first stained for RegI as described above. Following pretreatment with $1 \%$ normal goat serum in phosphate-buffered saline (PBS), the tissue sections were incubated with anti-chromogranin A polyclonal antibody for $2 \mathrm{~h}$ at room temperature. The sections were washed with PBS, then incubated with goat antirabbit IgG (Sigma) at room temperature for $30 \mathrm{~min}$. Bound chromogranin A-antibody complex was detected using an avidin-biotin peroxidase method (ABC Kit). Peroxidase activity was detected by incubating the samples with BCIP/ NBT solution (DAKO) for $10 \mathrm{~min}$ at room temperature, then counter staining with hematoxylin. After dehydration in a graded alcohol series, the sections were cleared in xylene.

\section{Formation of Gastric Mucosal Lesions Induced by Water-Immersion and Restraint Stress}

Adult male and female mice (9-15 week old) were used in the experiments. Before each experiment, animals were deprived of food but not water for $24 \mathrm{~h}$. The animals were then placed in restraint cages and immersed in a water bath $\left(25^{\circ} \mathrm{C}\right)$ to the level of the xiphoid process for $24 \mathrm{~h}$ as previously described. ${ }^{12,13}$ After the experiments, the animals were anesthetized and killed by cervical vertebral dislocation. Their stomachs were immediately removed and inflated with $2 \mathrm{ml}$ of $1 \%$ formalin solution for $10 \mathrm{~min}$. After opening along the greater curvature, gastric injury visible to the naked eye was found in the gastric mucosa as elongated black-red lines $(1-10 \mathrm{~mm}$ long by $0.5-1.5 \mathrm{~mm}$ wide) parallel to the long axis of the stomach in mice. The lines that resisted a strict washing by distilled water were distinguished from mere blood adhesion and were defined as erosions. The length (in millimeters) of each erosion was measured and cumulated as the ulcer index (UI) (in millimeters). This method for measuring mice and rats gastric ulcerative lesions was developed by Brodie and Hanson ${ }^{14}$ and has been widely used as a reliable method in many laboratories ${ }^{15-17}$ including ours. ${ }^{13}$

\section{Formation of Gastric Mucosal Lesions Induced by $\mathrm{HCL} /$ Ethanol}

The method is based on modifications of the method reported by Sun et al. ${ }^{18}$ Adult male and female mice (9-15 week old) were used. We checked the UI 6, 12, 18 and $24 \mathrm{~h}$ after $\mathrm{HCl} /$ ethanol injection to generate a time course. Before each experiment, animals were deprived of food and water for $24 \mathrm{~h}$. The animals received $0.2 \mathrm{ml}$ of $0.2 \mathrm{M} \mathrm{HCl} / 40 \%$ ethanol orally. The animals were killed at each time point and their stomachs were processed as described in the previous section to obtain the UI. 


\section{Isolation of Total RNA, RT and Real-Time Quantitative PCR}

For isolation of total RNA, the stomachs of Wt mice or Tg mice at the indicated time points were excised and stored in RNA later (Qiagen). Extraction of RNA from tissue samples stored in RNA later was performed by using the Qiagen RNeasy Mini kit (Qiagen) as described by the manufacturer. Extracted RNA (400 ng) was reverse transcribed into firststrand cDNA using the ProSTAR First-Strand RT-PCR Kit (Stratagene). Quantitative evaluation of $c-f o s, c-m y c$, rat regI, and $I L-1 \beta, T N F-\alpha$ and CXCL2 mRNA expression in the stomachs of Wt or Tg mice was performed by real-time quantitative PCR. $C$-fos cDNA was amplified with PCR primers $5^{\prime}$-ATCCGAAGGGAACGGAATAAG-3' and 5'-CAGACTTCTCATCTTCAAGTTGATCTG-3'; $C-m y c$ cDNA was amplified with the primers $5^{\prime}$-AGCTGTTTGAAGGCTGG ATTTC- $3^{\prime}$ and $5^{\prime}$-CTGTTGGTGAAGTTCACGTTGAG-3'; and rat RegI cDNA was amplified with the primers $5^{\prime}$ GGACACTGGGTATCCTAACAATTCC- $3^{\prime}$ and $5^{\prime}$ - CTCTCCA TTTCTTGTATCCTGAGTTTG- $3^{\prime}$. IL- $1 \beta$ cDNA was amplified with PCR primers $5^{\prime}$-AGGCTCCGAGATGAACAACA-3' and $5^{\prime}$-TTGGGATCCACACTCTCCA- ${ }^{\prime}$; TNF- $\alpha$ cDNA was amplified with PCR primers $5^{\prime}$-GAGGGGAATCCTTGG AAGA- $3^{\prime}$ and $5^{\prime}$-CTTGGGCCAGTGAGTGAAAG- $3^{\prime}$; and CXCL2 cDNA was amplified with PCR primers $5^{\prime}$-GCTGTC AATGCCTGAAGACC- $3^{\prime}$ and $5^{\prime}$-GGTCAGTTAGCCTTGCC TTT- $3^{\prime}$. As an internal control, mouse $\beta$-actin cDNA was amplified with the primers $5^{\prime}$-CGTGAAAAGATGACCCAG ATCA- ${ }^{\prime}$ and $5^{\prime}$-CACAGCCTGGATGGCTACGTA- $3^{\prime}$. Mouse GAPDH cDNA was amplified with the primers $5^{\prime}$-ACCCA GAAGACTGTGGATGG- $3^{\prime}$ and $5^{\prime}$-GGTCCTCAGTGTACC CAAG-3'. Real-time quantitative PCR was performed with an ABI PRISM 7700 Sequence Detection System using the SYBR Green PCR Master Mix and following the manufacturer's instructions (Applied Biosystems), except for the annealing temperature, which was $56^{\circ} \mathrm{C}$ for $20 \mathrm{~s}$ in our experiments. To exclude amplification of contaminating genomic DNA, all primers used in this study were carefully selected on different exons. We also included no-enzyme controls during RT, and no-template controls during PCR to check against possible contaminations. The relative expression levels of these genes, normalized against mouse $\beta$-actin expression or mouse GAPDH expression, were estimated with Q-Gene-analysis (Biotechnique Software, Westborough, MA, USA).

\section{Measurements of Serum Gastrin and Gastric Acid Secretion}

After deprivation of food for $24 \mathrm{~h}$, blood was collected by cardiac puncture. Serum was stored at $-20^{\circ} \mathrm{C}$ until assayed for gastrin (Gastrin-RIA kit II; Kyowa Medex, Tokyo, Japan). Basal gastric acid output (BAO) and secretagogue-stimulated gastric acid output (MAO) in Wt and $\mathrm{Tg}$ mice were examined with the pylorus-ligation method. After deprivation of food for $22 \mathrm{~h}$ and water for $2 \mathrm{~h}$ before experimentation, mice were subjected to epigastric laparotomy under light ether an- esthesia. The duodenum was identified and the junction between the pylorus and duodenum was picked up gently. The stomach itself was not disturbed. The pylorus was ligated with 4-0 silk, care being taken to avoid damage to blood vessels or traction on the stomach. The abdominal cavity was closed by suturing with 4-0 silk (BAO) or after intraperitoneal injection of gastrin (Gastrin I human; SigmaAldrich Japan, Tokyo, Japan), the volume of which depended on body weight. After $4 \mathrm{~h}$, stomachs were removed, and the gastric lumen was washed with $3.0 \mathrm{ml}$ of saline solution $(\mathrm{pH}$ 7.0). The gastric contents plus the washout solution were collected and centrifuged ( 3500 r.p.m., $10 \mathrm{~min}, 4^{\circ} \mathrm{C}$ ), then the volumes of supernatant and of solids were recorded. If the volume of solids was over $60 \mathrm{mg}$, the animal was discarded because of possible contamination of gastric contents with food residue or feces. Only the supernatant was used in the analysis. The acidity of the supernatant was determined by titration against $0.01 \mathrm{mM} \mathrm{NaOH}$ to $\mathrm{pH}$ 7.0. Gastric acid output (volume $\times$ acidity) was expressed as micromoles $\mathrm{H}^{+}$per $4 \mathrm{~h}$.

\section{Statistical Analysis}

The statistical significance of differences was evaluated with Student's $t$-test. A $P$-value $<0.05$ was considered to be significant.

\section{RESULTS \\ Normal Gastric Mucosa from Adult Wt Mice and Tg Mice Before Ulcer Induction}

In this study, we used 9- to 15-week-old adult littermate pairs for ulcer experiments. As a first step, we examined RegI expression and histological appearance of their stomachs before ulcer induction.

In the stomachs of the adult Wt mice, we were unable to detect any RegI-specific signals by immunohistochemistry, whereas a significant level of RegI expression was seen in the tissue of RegI-Tg mice (compare Wt control and Tg control in Figure 5a).

In spite of the difference of RegI expression, we found no histological difference in the gastric mucosa between Wt and $\mathrm{Tg}$ mice in the adult stage, in contrast to the infant stage ${ }^{8}$ in which $\mathrm{Tg}$ mice showed significant gastric hypertrophy and higher proliferating cell nuclear antigen expression compared with their Wt littermates. As shown in Figure 1a, HE staining of the fundic mucosa of 10-week-old Wt and Tg mice revealed no difference. In addition, the intensities and the locations of the proliferating cell nuclear antigen signals were almost the same in $\mathrm{Wt}$ and $\mathrm{Tg}$ mice (Figure $1 \mathrm{~b}$ ). To explore whether the difference between infant and adult could have resulted from a putative age-related decrease of transgene expression level, quantitative real-time RT-PCR was performed for the Tg regI gene. As shown in Figure 1c, there was no age-related decrease of the transgene, suggesting that the difference may be attributed in differences in tissue responsiveness to RegI. 
a

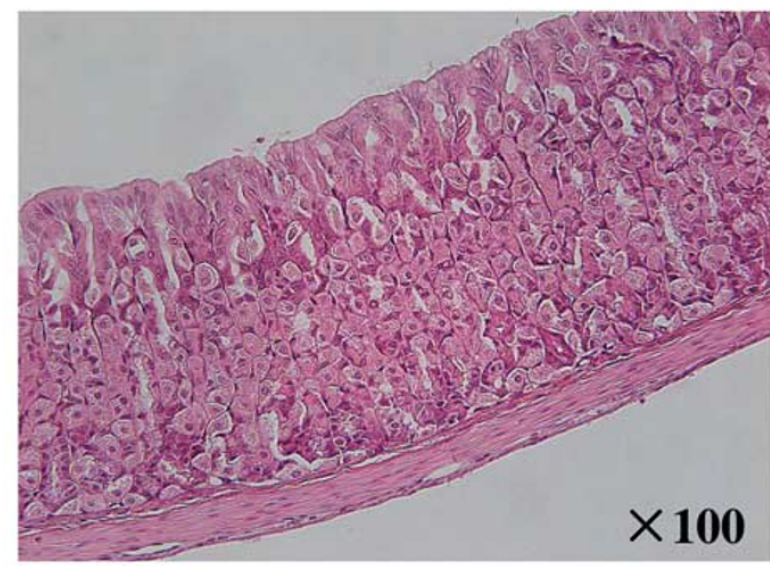

b

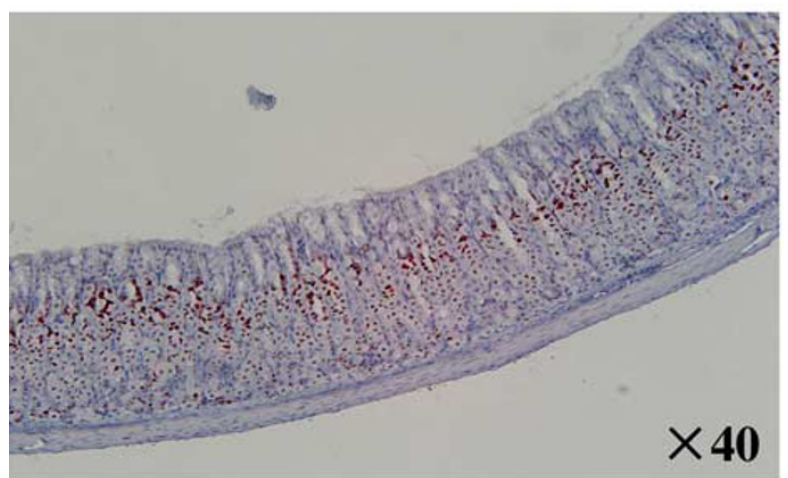

c

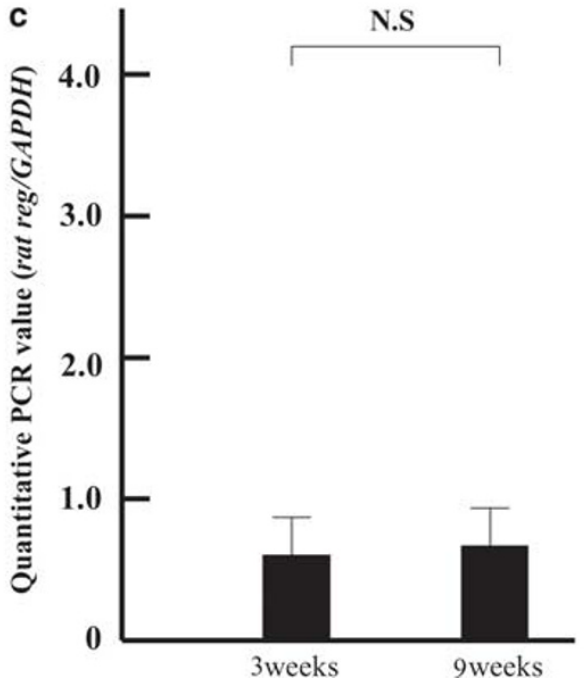

$\mathrm{Tg}$

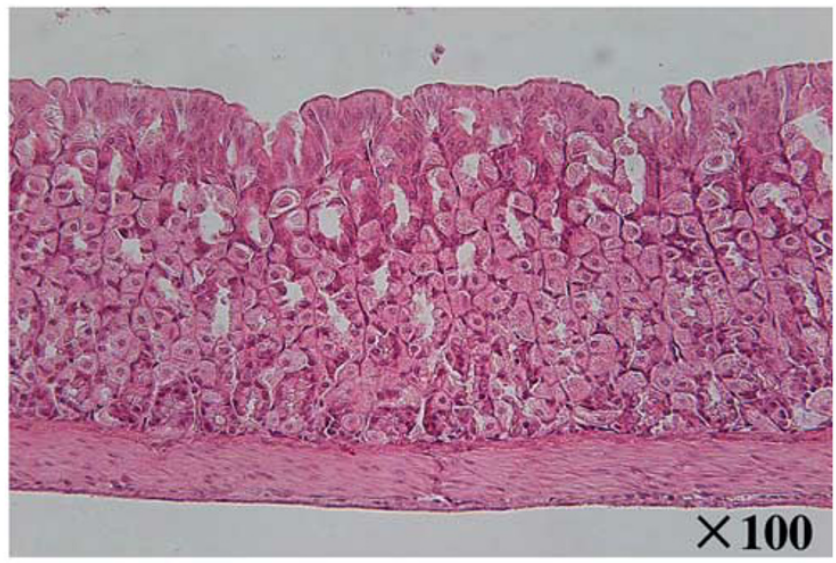

$\mathrm{Tg}$

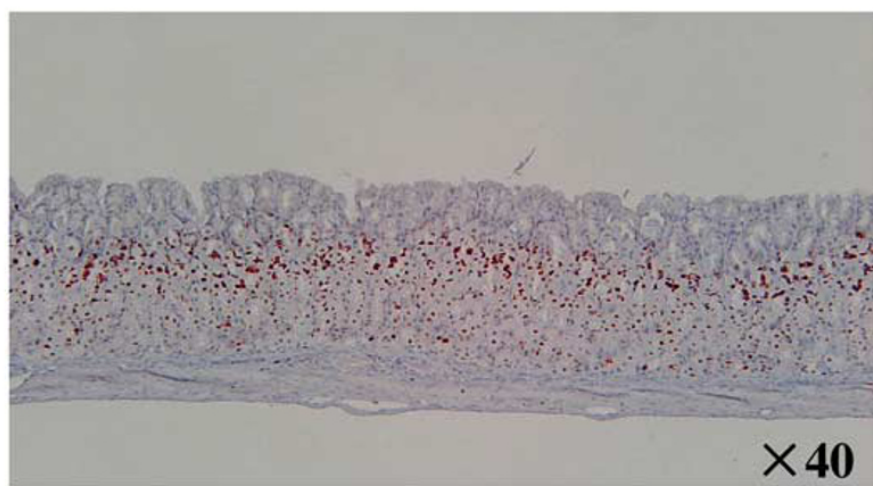

40

Figure 1 Morphological appearance of the stomach of adult transgenic $(\mathrm{Tg})$ mouse and wild-type $(\mathrm{Wt})$ littermate, and expression of the transgene (rat regl gene) at infant and adult stages without ulcer induction. (a) HE staining and (b) PCNA staining of the fundic mucosas of 10-week-old adult Tg mouse and Wt littermate. (c) Total RNA was extracted from stomachs of infant ( 3 weeks old) and adult (9 weeks old) Tg mice and analyzed by quantitative RT-PCR for expression of rat regl gene. Mean values \pm s.e.m. at each time point, normalized to the internal references (GAPDH). $N=10 \mathrm{for}$ infant mice, 7 for adult mice.

\section{Gastric Mucosal Lesions Induced by Water-Immersion Stress}

Adult $\mathrm{Tg}$ and Wt littermate pairs were subjected to waterimmersion stress and their stomachs were examined mac- roscopically for erosions. For all the littermate pairs tested, the UI of the Tg mice was less than that of the Wt mice at the end of water-immersion and restraint stress (Figure 2a). The mean index values were 8.3 for $\mathrm{Tg}$ mice and 23.7 for Wt mice, 
respectively (Figure 2a). Therefore, the transgene reduced the UI by two-thirds. To examine Tg regI gene (rat regI) expression in Tg mice, real-time RT-PCR was performed. In the Tg stomachs, expression of Tg regI gene was enhanced significantly after water-immersion stress (Figure $2 \mathrm{~b}$ ). In the Wt stomachs, no signal for endogenous mouse regI expression was detected in the same experiment, as the PCR primers were designed to anneal only to $\mathrm{Tg}$ rat sequence (data not shown).

\section{Gastric Mucosal Lesions Induced by HCL/Ethanol and the Time Course of Healing}

Adult $\mathrm{Tg}$ and $\mathrm{Wt}$ littermate pairs were given $\mathrm{HCl} /$ ethanol and examined for erosions at $6,12,18$ or $24 \mathrm{~h}$ after gavage. Almost all the littermate pairs (each linked by a thin dashed line in Figure 3) showed the superiority of Tg mice compared with Wt mice in the ability of decreasing UI (Figure 3a; right three columns), except for the pairs examined after $6 \mathrm{~h}$. Almost the opposite result was obtained for the $6 \mathrm{~h}$ pairs, in which $\mathrm{Tg}$ showed a higher index (Figure 3a; the leftmost column). The thick dotted line (Wt) and thick solid line (Tg) in Figure $3 \mathrm{a}$ show the time course of the average index values. In this experimental system, the injury phase seems to last around $6 \mathrm{~h}$ and the recovery phase should begin after $12 \mathrm{~h}$.
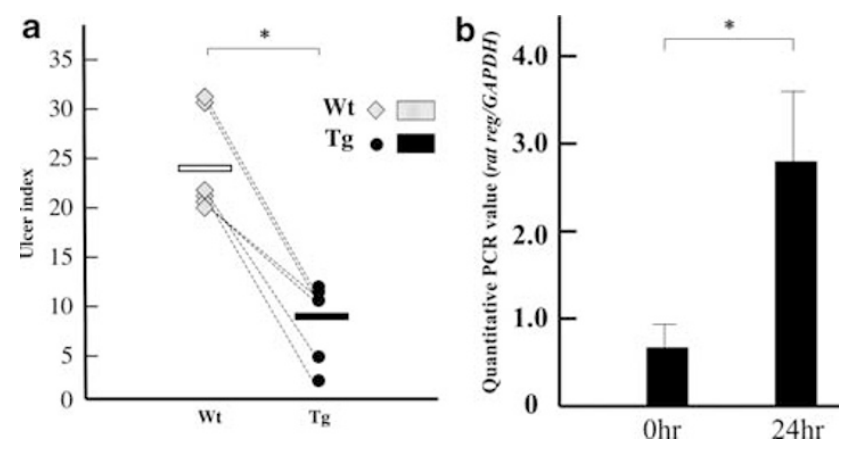

Figure 2 The ulcer index (UI) and expression of the transgene (rat regl gene) following exposure to water-immersion stress. (a) Adult gendermatched littermate pairs of $\mathrm{Wt}$ and $\mathrm{Tg}$ mice were exposed to $24 \mathrm{~h}$ of waterimmersion stress and their stomachs were scored for cumulative length of gastric erosions (UI). Values for each pair of littermates (each connected by thin dashed line) and mean values for Wt mice (stippled box) and Tg mice (filled box) are presented. $N=6$ pairs. ${ }^{*} P<0.05$. (b) Total RNA was extracted from adult Tg stomachs with $(24 \mathrm{~h})$ or without $(0 \mathrm{~h})$ water-immersion stress and was analyzed using quantitative RT-PCR for expression of rat regl gene. Mean values \pm s.e.m. at each time point, normalized to the internal references (GAPDH). $N=7$ for $0 \mathrm{~h}, 12$ for $24 \mathrm{~h}$. ${ }^{*} P<0.05$.
The mean UI value of Wt mice reached a maximum of 34.7 at $12 \mathrm{~h}$, then rapidly declined particularly between 12 and $18 \mathrm{~h}$. This decrease of index in Wt should be attributed to the upregulation of endogenous growth mediators, including RegI itself (see Figure 5a) and other factors. For Tg mice, a higher index was observed compared with that of Wt in the injury phase $(6 \mathrm{~h})$, indicating more severe injury. At $12 \mathrm{~h}$, the beginning of the recovery phase, this was reversed and $\mathrm{Tg}$ showed a lower index, suggesting faster initiation of the healing process. From this time point onward, Tg mice showed a significantly lower index, indicating a higher potential for ulcer healing. Figures $3 b$ and $c$ shows representative photographs of the stomachs from female littermate groups subjected to the time-course study.

\section{Time-Course Analysis of C-Fos and C-Myc Expression During Healing of the HCL/Ethanol Induced Lesion}

Using $c$-fos and $c$ - $m y c$ proto-oncogenes as an indicator of cell proliferation, ${ }^{19,20}$ we assessed the relative proportion of cells, which were proliferating in whole stomachs after treatment with $\mathrm{HCl} /$ ethanol in the above experiment (Figure 3), using real-time RT-PCR method. C-fos gene is an immediate early gene and its upregulation precedes cell proliferation. In the Wt mouse, $c$-fos gene expression reached a maximum at $6 \mathrm{~h}$, before the initiation of the recovery phase, then decreased gradually (Figure 4a). The temporal pattern of expression of c-fos gene in Tg stomach was similar; however, the expression levels were significantly higher in $\mathrm{Tg}$, especially at $12 \mathrm{~h}$ (Figure 4a). On the other hand, $c-m y c$ gene expression, which is known to be elevated at a later phase of cell proliferation, was expressed at slightly higher levels in Tg stomachs than in Wt stomachs particularly at 12 and $18 \mathrm{~h}$; however, the difference was not statistically significant (Figure $4 \mathrm{~b}$ ).

\section{Regl Expression During Healing of the HCL/Ethanol-Induced Lesions}

Expression of RegI protein in the stomachs was assessed by immunohistochemistry using a monoclonal antibody ${ }^{11}$ that equally recognizes rat (transgenic) and mouse (endogenous) RegI proteins. Before administration of $\mathrm{HCl} /$ ethanol, no RegI signal was detected in the gastric glands in the Wt stomachs (Figure 5a Wt control). After gastric lesions were induced, the signal appeared in the cells located around the ulcer beds (Figure 5a Wt HCl/ethanol), consistent with our previous observations $^{4}$ in the rat. In the case of $\mathrm{Tg}$ mouse, the control stomach showed higher signals compared with $\mathrm{Wt}$

Figure 3 The ulcer index (UI) following HCl/ethanol treatment. (a) Adult, gender-matched littermate pairs of Wt and Tg mice were orally administered $\mathrm{HCl}$ / ethanol, killed $6,12,18$ or $24 \mathrm{~h}$ after gavage and their stomachs were scored for cumulative length of gastric erosions (UI). Values for each pair of littermates (each connected by thin dashed line) and mean values at each time point for Wt mice (stippled boxes connected by thick dotted line) and Tg mice (filled boxes connected by thick solid line) are presented. Number and gender of experimental animals were as follows. For $6 \mathrm{~h}, n=9$ pairs (5 male pairs, 4 female pairs); for $12 \mathrm{~h}, n=12$ pairs ( 6 male pairs, 6 female pairs); for $18 \mathrm{~h}, n=11$ pairs ( 6 male pairs, 5 female pairs); for $24 \mathrm{~h}, n=10$ pairs ( 5 male pairs, 5 female pairs). ${ }^{\star} P<0.05$. (b) Representative macroscopic appearance of gastric mucosa at each time point (a group of female littermates). (c) Representative macroscopic appearance of gastric mucosa at $6 \mathrm{~h}$ examined at high magnification (female Wt mice). 


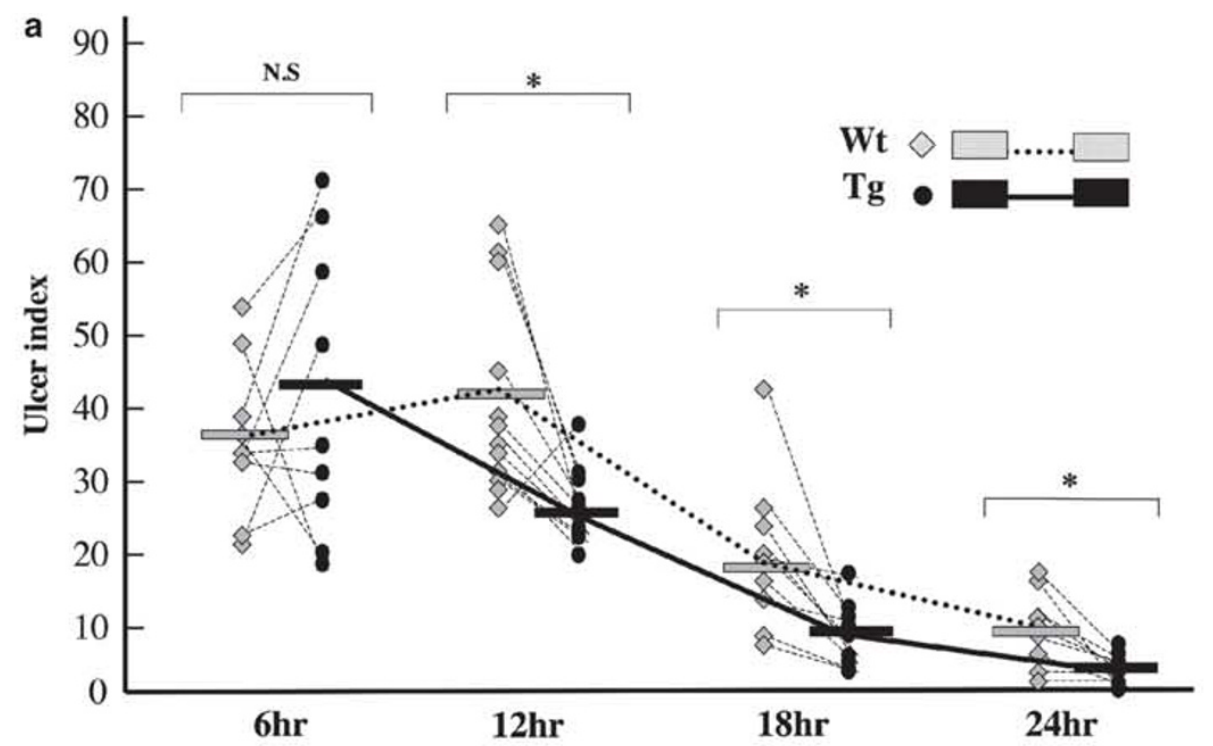

b

wild
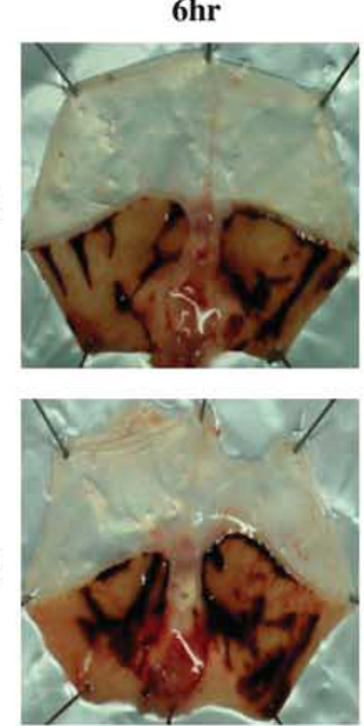

$12 \mathrm{hr}$
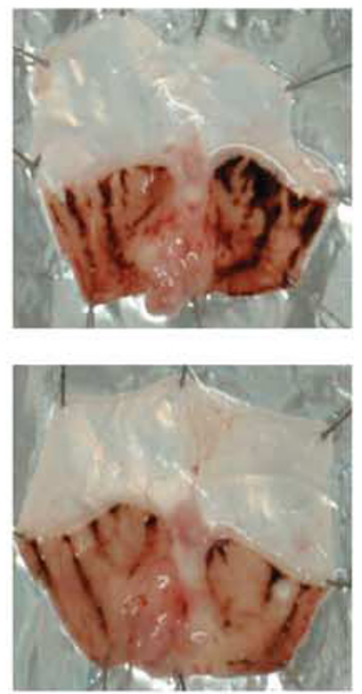

C
$18 \mathrm{hr}$
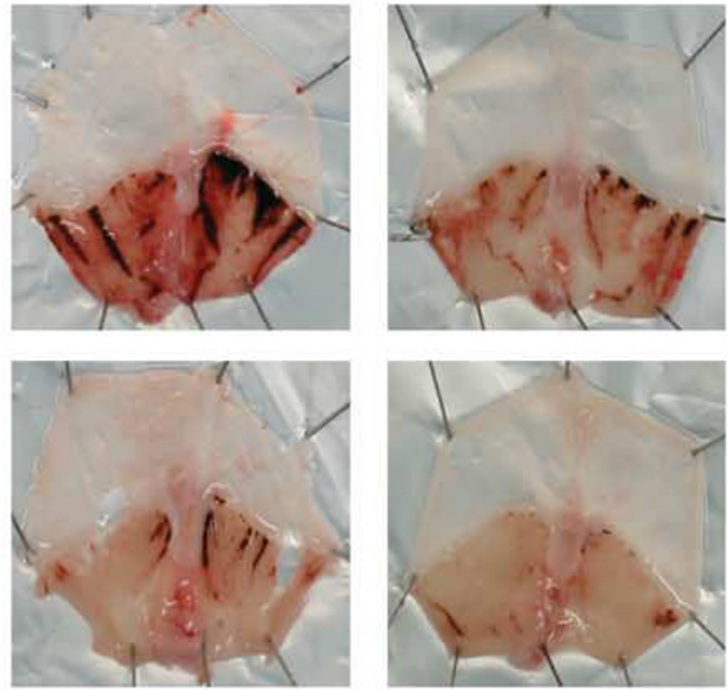

$24 \mathrm{hr}$

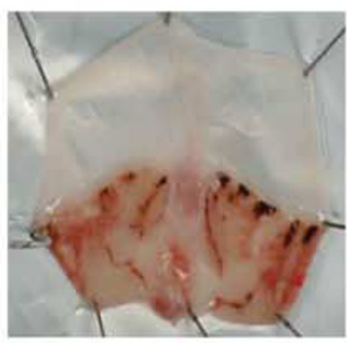

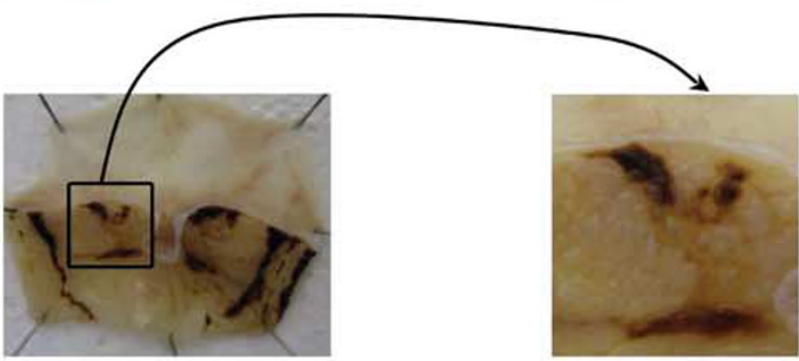

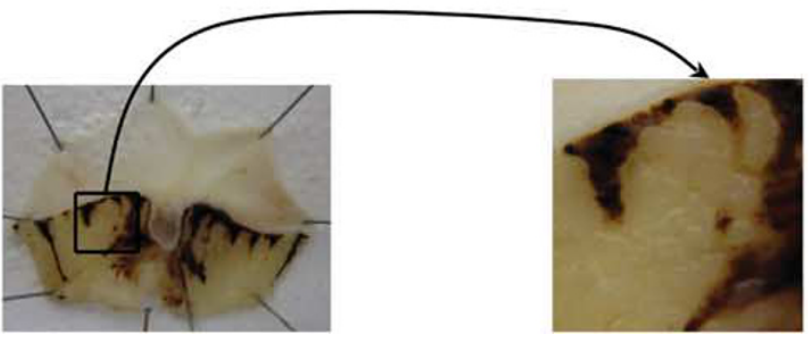



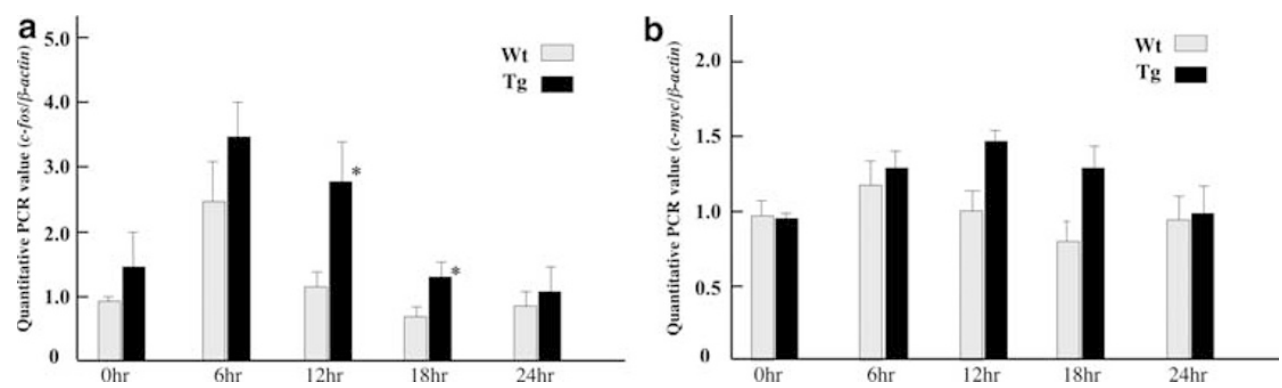

Figure 4 Expression levels of cell proliferation marker genes following the $\mathrm{HCl} /$ ethanol treatment. Total RNA was extracted from the stomachs of mice subjected to $\mathrm{HCl} /$ ethanol treatment and analyzed by quantitative RT-PCR for expression of $c$-fos and $c$-myc genes, general markers for cell proliferation. Mean values \pm s.e.m. at each time point for $c$-fos gene (a) and c-myc gene (b), normalized to the internal references $(\beta$-actin). $N=6$ pairs per time point. ${ }^{*}<0.05$.

(Figure 5a $\mathrm{Tg}$ control). Unexpectedly, $\mathrm{Tg}$ also showed elevated signal intensity after $\mathrm{HCl} / \mathrm{ethanol}$ gavage. Around the lesion induced in Tg stomach, still more intensive signals were seen than those in $\mathrm{Tg}$ control stomach (Figure $5 \mathrm{a} \mathrm{Tg}$ $\mathrm{HCl} /$ ethanol).

To examine the time course of Tg regI gene (rat regI) expression in Tg mice more quantitatively, real-time RT-PCR was used. In Tg stomachs, baseline expression of Tg regI gene was enhanced significantly $6 \mathrm{~h}$ after the $\mathrm{HCl} /$ ethanol gavage, then gradually decreased over the next $18 \mathrm{~h}$ to return to basal levels (Figure 5b). In the Wt stomachs, no signal for endogenous mouse regI expression was detected (data not shown), as was in the case of water-immersion stress experiment (Figure $2 \mathrm{~b}$ ). Using the same templates, the time course of several cytokines, which may be responsible for upregulation of regI gene, were examined. The cytokines, CXCL2, IL1- $\beta$ and TNF $\alpha$ were upregulated $6 \mathrm{~h}$ after the $\mathrm{HCl} /$ ethanol gavage (Figure $5 \mathrm{c}$ ).

Finally, the precise location of transgene expression in $\mathrm{Tg}$ stomachs before administration of $\mathrm{HCl} /$ ethanol was examined. Using sequential slice samples, location of the RegI signals was compared with that of signals of chromogranin A, a marker of endocrine cells. The chromogranin A signals were detected in the small cells located in the deep portion of the fundic grand, presumably indicating ECL cells (Figure 5d upper-left panel). On the other hand, the RegI signals were detected mainly in the cells of the same morphology and location (Figure 5d upper-right panel). These data suggest that the main site of expression for Tg RegI is ECL cell. Additional faint signals were seen for Tg RegI in other larger cell types, which may have morphological characteristics of chief cells (Figure 5d upper-right panel). Double staining for chromogranin A and Tg RegI was also performed (Figure 5d lower panel). The Tg RegI signals (brown) showed patchy distribution. In the RegI signal-negative glands, pure signals of chromogranin A (purple) were identified in small cells indicating ECL cells (white arrow). In the grand with RegI signals, double-stained (black) cell with morphological characteristics of ECL cell (black arrow) were identified, showing colocalization of chromogranin A and Tg RegI.
Gastric Acid Secretion and Serum Gastrin Concentration To assess gastric acid secretion, we examined the basal acid output (BAO) and maximum acid output (MAO) of adult Tg and Wt mice. As shown in Figure 6a, both BAO and MAO values of $\mathrm{Tg}$ mice were slightly higher than those of $\mathrm{Wt}$; however, there was no statistical difference. Also, there was no significant difference in serum gastrin concentration between $\mathrm{Tg}$ and Wt mice (Figure $6 \mathrm{~b}$ ).

\section{DISCUSSION}

Genetically engineered mice provide direct and compelling in vivo evidence for a function of a molecule. In this study, we induced experimental gastric ulcers in RegI-Tg mice and found that gastric ulcer healing was accelerated compared with their Wt littermates. This is the first in vivo evidence that RegI contributes to gastric ulcer healing. To our knowledge, among epithelial growth factors, only trefoil factor had been shown by this strategy to be involved in ulcer healing. ${ }^{21}$ From this point of view, RegI is the second factor to be identified. It will be interesting to clarify in future studies the exact role each factor has in the process of ulcer healing. We used two experimental systems based on different theories to induce ulcer formation (stress-induced and $\mathrm{HCl} /$ ethanol-induced) and used a large battery of littermate pairs to reach a definitive conclusion. In both experimental systems and in almost all the littermate pairs tested (with the only exception of the $6 \mathrm{~h}$ group in $\mathrm{HCl} /$ ethanol experiment), $\mathrm{Tg}$ mice showed lower ulcer indices than Wt (Figures 2 and 3), clearly showing the strong potential of RegI for ulcer healing.

It is important to clarify whether RegI contributes to healing by a cytoprotective mechanism or promotion of growth following physical injury. Several findings in this study favor the latter possibility. First, RegI was shown to exhibit its effect in the recovery phase rather than in the injury phase. Using a large array of littermate pairs, the time courses of ulcer healing in both Wt and $\mathrm{Tg}$ mice were precisely delineated and compared (Figure $3 \mathrm{a}$ ). In the injury phase, the mean index value for $\mathrm{Tg}$ was higher than that for Wt. This was reversed in the recovery phase, when Tg showed 
a
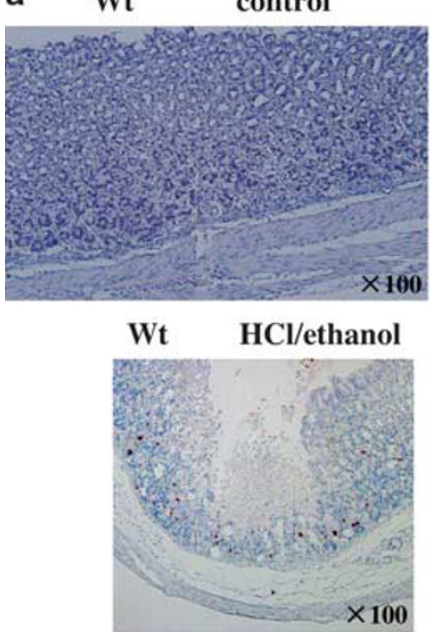

$\mathrm{Tg}$
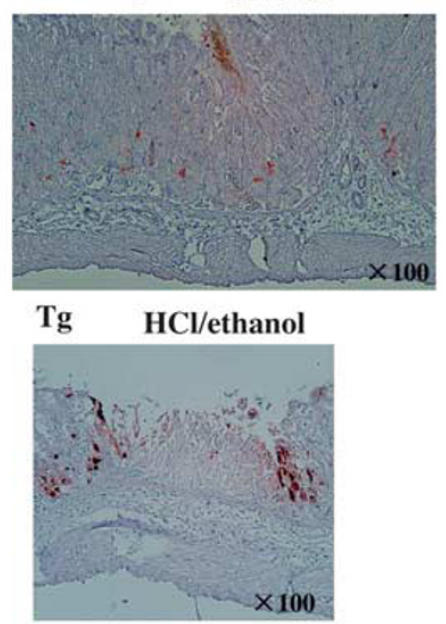

b

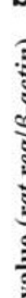

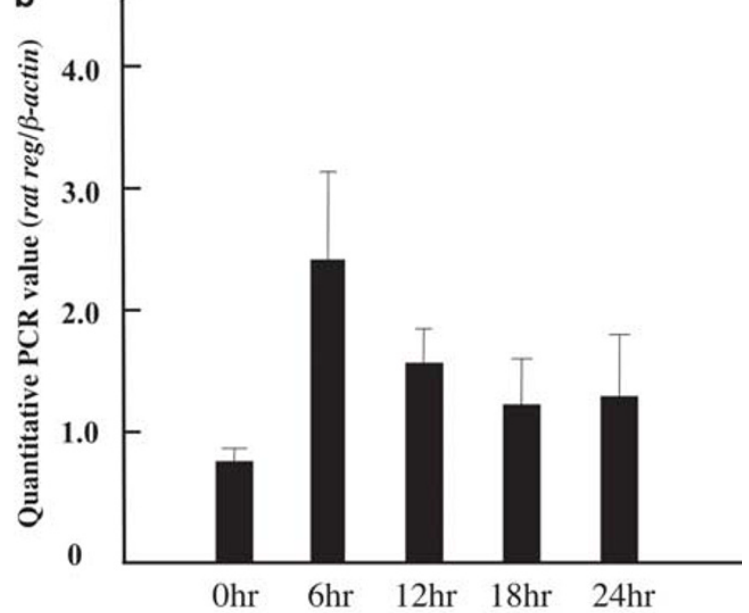

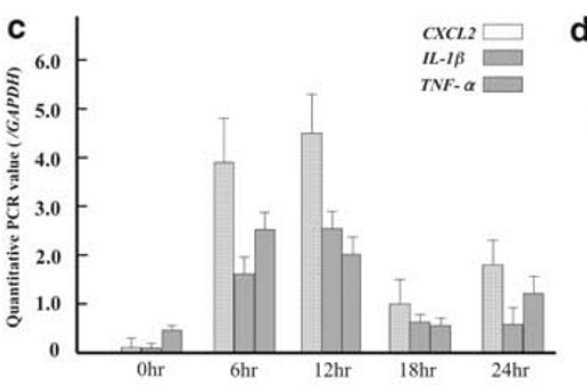
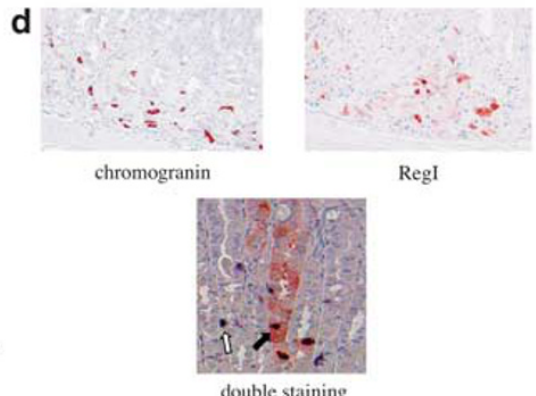

double staining

Figure 5 Expression of Regl and various cytokines before and after $\mathrm{HCl} /$ ethanol treatment. (a) Stomachs were obtained before (control) and $6 \mathrm{~h}$ after $\mathrm{HCl} /$ ethanol administration. Tissue was fixed and analyzed by immunohistochemistry using a monoclonal antibody, ${ }^{11}$ which recognizes transgenic and endogenous Regl proteins to equal extent. (b) Total RNA was extracted from Tg stomachs subjected to $\mathrm{HCl} /$ ethanol treatment and analyzed by quantitative RT-PCR for expression of the rat regl gene (transgene). Mean values \pm s.e.m. at each time point, normalized to the internal references ( $\beta$-actin). $N=6$ pairs per time point. (c) Total RNA was extracted from Tg stomachs subjected to HCl/ethanol treatment and analyzed by quantitative RT-PCR for expression of $C X C L 2, I L 1-\beta$ or TNF- $\alpha$. Mean values \pm s.e.m. at each time point, normalized to the internal references (GAPDH). $N=6$ per time point. (d) The Tg stomachs before $\mathrm{HCl}$ /ethanol administration were stained with anti-chromogranin A antibody (upper-left panel) and the anti-Regl antibody (upper-right panel). The lower panel shows the results of immunohistochemical double staining for chromogranin A (purple) and Regl (brown). The white arrow indicates a cell with pure chromogranin A signal (purple) and the black arrow indicates a cell with mixed signal (black).
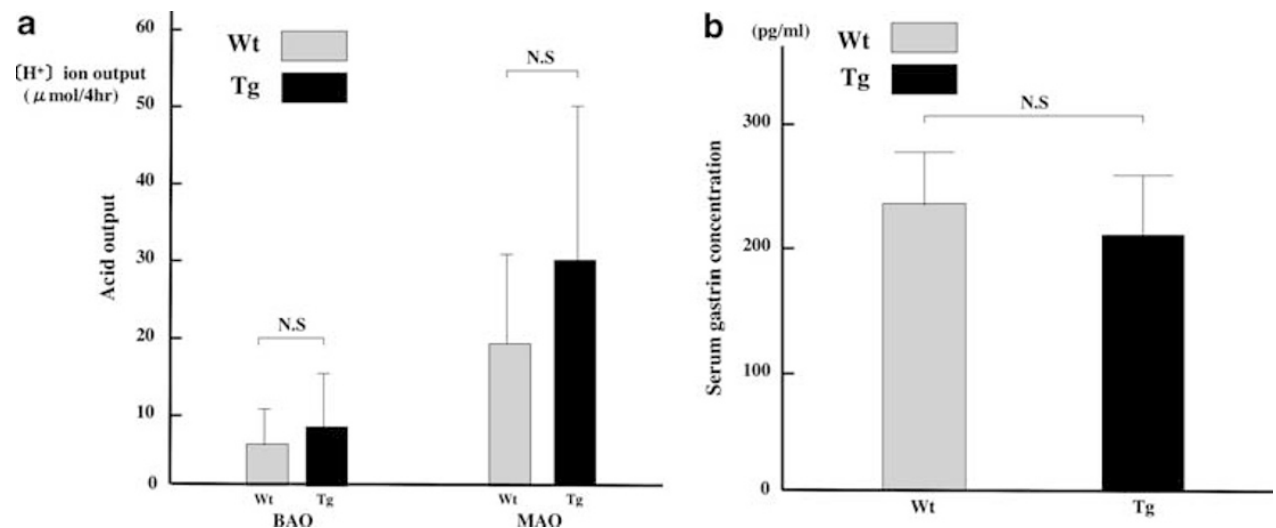

Figure 6 Basal acid output (BAO), maximum acid output (MAO) and serum gastrin concentration in Wt and Tg mice. (a) Adult, gender-matched littermate pairs of Wt and Tg mice without water-immersion stress or $\mathrm{HCl} /$ ethanol gavage were examined with the pylorus-ligation method for BAO ( $n=7$ pairs) and secretagogue-stimulated gastric acid output (MAO) ( $n=8$ pairs). Results are expressed as mean values \pm 1 s.d. (b) Serum from adult, gender-matched littermate pairs of Wt and Tg mice without water-immersion stress or $\mathrm{HCl} /$ ethanol gavage were examined for gastrin concentration ( $n=6$ pairs). Results are expressed as mean values \pm 1 s.d. 
constant superiority in healing over Wt (Figure 3a). Generally speaking, the recovery phase corresponds to the phase of progenitor cells proliferation, when the cells divide, migrate and differentiate to reconstruct the gastric tissue architecture. As RegI was effective in the recovery phase rather than in the injury phase, it is reasonable to speculate that RegI participates in ulcer healing by stimulating progenitor cell growth. It is unclear why the UI was higher in Tg mice in the early injury phase $(6 \mathrm{~h})$. No matter the reason, the data rather argue against RegI-mediated cytoprotection as the underlying mechanism promoting gastric healing. If RegI had used a cytoprotective mechanism, the UI of Tg mice would have been lower than in Wt mice from early in the injury phase. Second, at least, we ruled out the possibility that RegI inhibits gastric acid secretion to contribute to the cytoprotection mechanism. Some other factors, for example epidermal growth factor, have been shown to act as cytoprotective agents by virtue of their inhibitory effects on gastric acid secretion..$^{22,23}$ Our current data for gastric acid output did not reveal inhibitory activity by RegI (Figure 6). Third, expression of $c$-fos gene, which was used as proliferation markers, was significantly higher in $\mathrm{Tg}$ than in $\mathrm{Wt}$ mice in the time course following $\mathrm{HCl} /$ ethanol-induced injury. Throughout the time course, the gene showed higher expression levels in $\mathrm{Tg}$ mice (Figures $4 \mathrm{a}$ and $\mathrm{b}$ ). The increase in $c$-fos gene expression (which is known to precede cell proliferation) was prominent at the beginning of the healing phase (Figure 4a). These data show that the fraction of proliferating cells increased in $\mathrm{HCl} /$ ethanol-treated $\mathrm{Tg}$ mice in the course of ulcer healing and support the hypothesis that RegI uses a growth-promoting mechanism to contribute to ulcer healing.

We examined the temporal expression pattern of RegI in the $\mathrm{HCl} /$ ethanol-treated mice. We found that the expression of the transgene in the stomachs of $\mathrm{Tg}$ mice was upregulated in response to $\mathrm{HCl} /$ ethanol administration, as well as the expression of the endogenous regI gene in the stomachs of Wt mice (Figure 5). This was an unexpected result because transgene expression was driven by an artificial promoter based on a human CMV (hCMV) promoter sequence, not by the endogenous regI promoter that responds to inflammatory stimuli. However, we found a clear answer to this issue in previous reports. $^{24,25}$ Those reports described the responsiveness of the hCMV promoter to various inflammatory cytokines including IL-1 $\beta$ and TNF- $\alpha$. In addition, it is reported that those cytokines are produced from inflammatory sites, functioning as inflammatory mediators in the process of gastric ulcer healing. ${ }^{13,26,27,28}$ Taken together, it is reasonable to speculate that these inflammatory cytokines were secreted from the site of the injury and delivered to the cells located around the ulcerated craters, resulting in the activation of the Tg promoter (hCMV promoter). We confirmed that these cytokine genes are indeed upregulated in Tg stomachs in response to $\mathrm{HCl} /$ ethanol administration (Figure $5 c)$. We also examined spatial expression pattern of RegI in
Tg stomach. Unexpectedly again, the Tg RegI expression was mainly directed to the ECL cells, which is the site of expression for endogenous RegI (Figure 5d). The mechanism for this is unclear and should be clarified in future studies. We speculate that there may be a post-transcriptional control mechanism specific to ECL cells, by which the regI mRNA molecule is efficiently translated into RegI protein.

In spite of the different RegI expression levels in Wt and $\mathrm{Tg}$ adult (10 weeks old) mice (compare Figure 5a Wt control and Tg control), there was no detectable morphological difference between their gastric tissues (Figures $1 \mathrm{a}$ and $\mathrm{b}$ ). This was advantageous to our investigation because morphological differences would have obscured the function of RegI in ulcer healing. It is unclear why RegI showed its effect only in the infant stage (3-week stage of gastric gland morphogenesis) ${ }^{8}$ and in the ulcer-healing stage, but did not in the adult stage. It is possible that RegI has specific roles in stages of generation (infant) and regeneration (ulcer healing) of the gastric tissue when the tissue is being constructed from 'nothing', and has no role in the stage when the constructed tissue structure is maintained by cell turnover (adult). However, this is only a speculation with no observational support. Difference of the nature of the progenitor cells between these stages must be demonstrated in the future study, focusing particularly on the molecular basis of RegI cell surface receptor function.

It is also unclear how RegI exerts proliferative effects on gastric cells. Tritiated thymidine/EM autoradiographic lineage-tracing studies have delineated the morphological features of the stem cell niche in adult mouse stomach. ${ }^{29}$ According to these studies, the isthmus part of the gastric gland seems to harbor gastric multipotent stem cells and their committed daughters (progenitor cells), prepit cell precursors, preneck cell precursor and parietal cell progenitors. On the other hand, in our previous study, ${ }^{8}$ there was an increase in both the number and staining intensity of proliferation-associated markers in the isthmus region of the RegI-Tg mouse. These data suggest that stem cells and/or committed progenitor cells are the targets of RegI function. However, at this time, there is no information about which class of early cells is the direct target of RegI. This issue is important because the gastric cell population was biased to chief cells and parietal cell lineages in the RegI-Tg mouse. ${ }^{8}$ This bias is easily explained if RegI targeted specific fractions of committed progenitor cells, namely, preneck cell precursors and parietal cell progenitors, which are the origins of chief cells and parietal cells, respectively. In this hypothesis, prepit cell precursors, which are the precursors of pit cells, do not respond to a RegI signal. If, on the contrary, RegI targeted multipotent stem cells, a more interesting but complicated model must be formulated. In this case, RegI likely engages an intracellular signaling pathway that induces asymmetric stem cell division simultaneously with directing differentiation to preneck cell precursors and parietal cell progenitors. This issue needs to be clarified through detailed future stud- 
ies of the RegI receptor expression pattern among stem cells and committed progenitor cells, and through delineation of downstream signaling pathways.

\section{DISCLOSURE/CONFLICT OF INTEREST}

The authors declare no conflict of interest.

1. Tarnawski AS. Cellular and molecular mechanisms of gastrointestinal ulcer healing. Dig Dis Sci 2005;50(Suppl 1):S24-S33.

2. Wong WM, Playford RJ, Wright NA. Peptide gene expression in gastrointestinal mucosal ulceration: ordered sequence or redundancy? Gut 2000;46:286-292.

3. Terazono K, Yamamoto $\mathrm{H}$, Takasawa S, et al. A novel gene activated in regenerating islets. J Biol Chem 1988;263:2111-2114.

4. Asahara $M$, Mushiake $S$, Shimada $S$, et al. Reg gene expression is increased in rat gastric enterochromaffin-like cells following water immersion stress. Gastroenterology 1996;111:45-55.

5. Fukui $H$, Kinoshita $Y$, Maekawa $T$, et al. Regenerating gene protein may mediate gastric mucosal proliferation induced by hypergastrinemia in rats. Gastroenterology 1998;115:1483-1493.

6. Kazumori $\mathrm{H}$, Ishihara $\mathrm{S}$, Hoshino $\mathrm{E}$, et al. Neutrophil chemoattractant 2 beta regulates expression of the Reg gene in injured gastric mucosa in rats. Gastroenterology 2000;119:1610-1622.

7. Yoshino $\mathrm{N}$, Ishihara $\mathrm{S}$, Rumi $\mathrm{MA}$, et al. Interleukin-8 regulates expression of Reg protein in Helicobacter pylori-infected gastric mucosa. Am J Gastroenterol 2005;100:2157-2166.

8. Miyaoka $Y$, Kadowaki $Y$, Ishihara $S$, et al. Transgenic overexpression of Reg protein caused gastric cell proliferation and differentiation along parietal cell and chief cell lineages. Oncogene 2004;23:3572-3579.

9. Ose $\mathrm{T}$, Kadowaki $\mathrm{Y}$, Fukuhara $\mathrm{H}$, et al. Reg I-knockout mice reveal its role in regulation of cell growth that is required in generation and maintenance of the villous structure of small intestine. Oncogene 2007:26:349-359.

10. Unno $M$, Yonekura $H$, Nakagawara $K$, et al. Structure, chromosomal localization, and expression of mouse reg genes, reg I and reg II. A novel type of reg gene, reg II, exists in the mouse genome. J Biol Chem 1993;268:15974-15982.

11. Terazono $\mathrm{K}$, Uchiyama $\mathrm{Y}$, Ide $\mathrm{M}$, et al. Expression of reg protein in rat regenerating islets and its co-localization with insulin in the beta cell secretory granules. Diabetologia 1990;33:250-252.

12. Takagi $K$, Okabe $S$. The effects of drugs on the production and recovery processes of the stress ulcer. Jpn J Pharmacol 1968;18:9-18.

13. Matsushima $Y$, Kinoshita $Y$, Watanabe $M$, et al. Augmentation of waterimmersion stress-induced gastric mucosal lesions in BALB/c mice infected with Helicobacter felis. Digestion 1999;60:34-40.
14. Brodie DA, Hanson HW. A study of the factors involved in the production of gastric ulcers by restraint technique. Gastroenterology 1960;38:353-361.

15. Cho $\mathrm{CH}, \mathrm{Wu} \mathrm{KK}, \mathrm{Wu} \mathrm{S}$, et al. Morphine as a drug for stress ulcer prevention and healing in the stomach. Eur J Pharmacol 2003;460: 177-182.

16. Odashima $M$, Otaka $M$, Jin $M$, et al. Selective adenosine $A_{2 A}$ receptor agonist, ATL-146e, attenuates stress-induced gastric lesions in rats. J Gastroenterol Hepatol 2005;20:275-280.

17. Arun M, Asha VV. Gastroprotective effect of Dodonaea viscose on various experimental ulcer models. J Ethnopharmacology 2008:118:460-465.

18. Sun XB, Matsumoto T, Yamada H. Effects of a polysaccharide fraction from the roots of Bupleurum falcatum $L$. on experimental gastric ulcer models in rats and mice. J Pharm Pharmacol 1991;43:699-704.

19. Yoshiura K, Ota S, Terano A, et al. Growth regulation of rabbit gastric epithelial cells and proto-oncogene expression. Dig Dis Sci 1994:39:1454-1463.

20. Ito T, Hayashi N, Sasaki Y, et al. Sequential proto-oncogene expression during regeneration in rat stomach. Gastroenterology 1990;98: 1525-1531.

21. Farrell JJ, Taupin D, Koh TJ, et al. TFF2/SP-deficient mice show decreased gastric proliferation, increased acid secretion, and increased susceptibility to NSAID injury. J Clin Invest 2002;109:193-204.

22. Gregory $\mathrm{H}$. Isolation and structure of urogastrone and its relationship to epidermal growth factor. Nature 1975;257:325-327.

23. Finke $U$, Rutten $M$, Murphy RA, et al. Effects of epidermal growth factor on acid secretion from guinea pig gastric mucosa: in vitro analysis. Gastroenterology 1985;88:1175-1182.

24. Kline JN, Hunninghake GM, He B, et al. Synergistic activation of the human cytomegalovirus major immediate early promoter by prostaglandin E2 and cytokines. Exp Lung Res 1998;24:3-14.

25. Ritter T, Brandt $C$, Prösch S, et al. Stimulatory and inhibitory action of cytokines on the regulation of hCMV-IE promoter activity in human endothelial cells. Cytokine 2000;12:1163-1170.

26. Tamura M, Arakaki N, Tsubouchi $\mathrm{H}$, et al. Enhancement of human hepatocyte growth factor production by interleukin-1 alpha and -1 beta and tumor necrosis factor-alpha by fibroblasts in culture. J Biol Chem 1993;268:8140-8145.

27. Kinoshita $Y$, Nakata $H$, Hassan $\mathrm{S}$, et al. Gene expression of keratinocyte and hepatocyte growth factors during the healing of rat gastric mucosal lesions. Gastroenterology 1995;109:1068-1077.

28. Wallace $\mathrm{JL}$, Chin BC. Inflammatory mediators in gastrointestinal defense and injury. Proc Soc Exp Biol Med 1997;214:192-203.

29. Karam SM, Leblond CP. Dynamics of epithelial cells in the corpus of the mouse stomach. I. Identification of proliferative cell types and pinpointing of the stem cell. Anat Rec 1993;236:259-279. 